\title{
TiBuana
}

\section{Anthropometry Application for Flexible Chair College}

\author{
Andarmadi JatiAbdhi Wasesa ${ }^{1}$, Muhamad Abdul Jumali ${ }^{2}$ \\ ${ }^{1,2}$ Industrial Engineering Department, Engineering Faculty, University of PGRI Adi Buana \\ Email: andarmadi@unipasby.ac.id
}

\begin{abstract}
Anthropometry is a part of ergonomics that specifically studies body size which includes linear dimensions, as well as content, also includes areas of size, strength, speed, other aspects of body movement. Chairs are furniture that functions as seats, with the demands of the public for the design of chairs, many innovations have sprung up for the manufacture of chairs, the comfort factor is the most served in the process of making chairs, one of which is the lecture chair. The research subjects in this study were undergraduate students at a tertiary institution in East Java with male and female sex, with an age range of 18-25 years. As a result, for Hips Width The size of the seat width used is $36 \mathrm{~cm}$ (95th percentile) so that large people can fit when occupying a chair, for Back Height The height of the chair is $57 \mathrm{~cm}$ (5th percentile) so that people with small body postures can sit comfortably without difficulty, for Knee Length The length of the knee is $61 \mathrm{~cm}$ (95th percentile).
\end{abstract}

Keywords: anthropometry, lecture chairs, hips, back height, knee-length.

\section{INTRODUCTION}

According to Stevenson (1989), anthropometry is a collection of numerical data related to the physical characteristics of the human body, size, shape, and strength and the application of these data to address design problems. Anthropometry can be divided into two which are Static Anthropometry and Dynamic Anthropometry. Static anthropometry is the size of the body and the characteristics of the body at rest (static) for a predetermined or standard position, for example, Height, Shoulder width.Dynamic anthropometry is body size or body characteristics in motion or paying attention to movements that may occur when the worker carries out activities., for example, The angle of the hand, the angle of the ankle. In the design used anthropometric data, three principles must be considered, namely (Wignjosoebroto, 2003): The principle of designing facilities based on extreme individuals (minimum or maximum) and Customizable facility design principle Chairs are furniture that functions as seats, with the demands of the public for the design of chairs, many innovations have sprung up for the manufacture of chairs, the comfort factor is the most served in the process of making chairs, one of which is the lecture chair. Putri (2014) suggests that from the results of anthropometric measurements that are compared with the results of measuring the dimensions of tables and chairs, as well as strengthened by the activity shooting data from research subjects, it is concluded that there is a relationship between the size of an ergonomic table and chair with the comfort of children at school.

\section{METHODOLOGY}

The research subjects in this study were undergraduate students at a tertiary institution in East Java with male and female sex, with an age range of 18-25 years.

Anthropometric data collection is carried out to obtain ergonomic conditions in the design of the latest lecture chair designs. The use of anthropometric data is carried out so that the designed product can be adjusted to the user's body. The following are the stages in anthropometry, Establish design requirements, Determine and describe the user population, Selection of samples to be taken data, Determination of data requirements (body dimensions to be taken), Determination of data sources (body dimensions to be taken) and selection of percentiles to beused, Preparation of measuring instruments to be 
DOI : https://doi.org/10.36456/tibuana.4.01.3175.1-6

used, Retrieval of data, Data processing, Visualize.

Some of the data processing carried out on anthropometric data are:

a. Data Adequacy Test

$$
N^{\prime}=\left\lfloor\frac{\frac{k}{s} \sqrt{N \sum x^{2}-}\left(\sum x\right) 2}{\sum x}\right\rfloor
$$

Where: $\mathrm{k}=$ Confidence Level (If the confidence level is $99 \%$, then $\mathrm{k}=2.58 \approx 3$, If the confidence level is $95 \%$, then $\mathrm{k}=1.96 \approx 2$, If the confidence level is $68 \%$, then $\mathrm{k} \approx 1$ ) $\mathrm{s}=$ Degree of Accuracy

$e$-ISSN 2622-2035

\section{RESULTS AND DISCUSSION}

$\mathrm{N}=$ Total Observation Data

$\mathrm{N}^{\prime}=$ Total Theoretical Data

$\mathrm{x}=$ Observation Data

If $\mathrm{N}$ ' $\leq \mathrm{N}$ then the data is considered sufficient, but if $\mathrm{N}^{\prime}>\mathrm{N}$ data is insufficient (insufficient) and it is necessary to add data. By using the formula above, the results of the data adequacy test will be obtained from each dimension of the body.

Table 1. Anthropometri Data

\begin{tabular}{|c|c|c|}
\hline HIP WIDTH & SHOULDER HEIGHT & KNEE LENGTH \\
\hline 34 & 61 & 56 \\
\hline 34 & 62 & 57 \\
\hline 35 & 63 & 51 \\
\hline 31 & 57 & 42 \\
\hline 33 & 60 & 51 \\
\hline 34 & 61 & 55 \\
\hline 36 & 65 & 54 \\
\hline 34 & 61 & 54 \\
\hline 32 & 58 & 45 \\
\hline 34 & 61 & 53 \\
\hline 33 & 60 & 50 \\
\hline 34 & 61 & 53 \\
\hline 31 & 57 & 42 \\
\hline 34 & 62 & 56 \\
\hline 35 & 62 & 58 \\
\hline 34 & 61 & 55 \\
\hline 34 & 61 & 53 \\
\hline 30 & 54 & 35 \\
\hline 33 & 60 & 52 \\
\hline 35 & 63 & 60 \\
\hline 34 & 61 & 47 \\
\hline 33 & 59 & 49 \\
\hline 35 & 63 & 59 \\
\hline 33 & 60 & 51 \\
\hline 33 & 60 & 50 \\
\hline 35 & 63 & 60 \\
\hline 32 & 58 & 45 \\
\hline 34 & 61 & 53 \\
\hline 33 & 60 & 51 \\
\hline 33 & 60 & 52 \\
\hline 33 & 60 & 52 \\
\hline 34 & 61 & 55 \\
\hline 34 & 61 & 55 \\
\hline 32 & 59 & 47 \\
\hline
\end{tabular}


TiBuana

Journal of applied Industrial Engineering-University of PGRI AdiBuana

DOI : https://doi.org/10.36456/tibuana.4.01.3175.1-6

$p$-ISSN 2622-2027

$e$-ISSN 2622-2035

2| TiBuana, Vol. 04, No.1, 2021 
Table 2. Determine the width of the hips

\begin{tabular}{|c|c|c|c|c|c|c|}
\hline $\mathrm{NO}$ & NAME & HIP WIDTH & $\mathrm{F}$ & $\mathrm{FX}$ & DEVIATION EACH SCORE (X) & $X^{2}$ \\
\hline 1 & Rindra & 34 & 1 & 34 & 0.52 & 0.28 \\
\hline 2 & M.Rizki & 34 & 1 & 34 & 0.52 & 0.28 \\
\hline 3 & Nanda & 35 & 1 & 35 & 1,52 & 2,33 \\
\hline 4 & Rida & 31 & 1 & 31 & -2.47 & 6.10 \\
\hline 5 & Alfin & 33 & 1 & 33 & -0.47 & 0.22 \\
\hline 6 & Septiyan & 34 & 1 & 34 & 0.52 & 0.28 \\
\hline 7 & Karto & 36 & 1 & 36 & 2.52 & 6.39 \\
\hline 8 & Fani & 34 & 1 & 34 & 0.52 & 0.28 \\
\hline 9 & Opik & 32 & 1 & 32 & -1.47 & 2.16 \\
\hline 10 & Eko & 34 & 1 & 34 & 0.52 & 0.28 \\
\hline 11 & Hendy & 33 & 1 & 33 & -0.47 & 0.22 \\
\hline 12 & Dennis & 34 & 1 & 34 & 0.52 & 0.88 \\
\hline 13 & Titin & 31 & 1 & 31 & -2.47 & 6.10 \\
\hline 14 & Dirga & 34 & 1 & 34 & 0.52 & 0.28 \\
\hline 15 & Akhmad & 35 & 1 & 35 & 1.52 & 2.33 \\
\hline 16 & M. Jafar & 34 & 1 & 34 & 0.52 & 0.28 \\
\hline 17 & M. Hanif & 34 & 1 & 34 & 0.52 & 0.28 \\
\hline 18 & Elsa & 30 & 1 & 30 & -3.47 & 12.04 \\
\hline 19 & Febian & 33 & 1 & 33 & -0.47 & 0.22 \\
\hline 20 & M.Abid & 35 & 1 & 35 & 1.52 & 2.33 \\
\hline 21 & Widodo & 34 & 1 & 34 & 0.52 & 0.28 \\
\hline 22 & Rahmatulloh & 33 & 1 & 33 & -0.47 & 0.22 \\
\hline 23 & Nova & 35 & 1 & 35 & 1.52 & 2.33 \\
\hline 24 & Candra & 35 & 1 & 35 & -0.47 & 0.22 \\
\hline 25 & Alex & 33 & 1 & 33 & -0.47 & 0.22 \\
\hline 26 & Prananda & 33 & 1 & 33 & 1.52 & 2.33 \\
\hline 27 & Gunawan & 35 & 1 & 35 & -1.47 & 2.16 \\
\hline 28 & Prayoga & 32 & 1 & 32 & 0.52 & 0.28 \\
\hline 29 & Rony & 34 & 1 & 34 & -0.47 & 0.22 \\
\hline 30 & Iwan & 33 & 1 & 33 & -0.47 & 0.22 \\
\hline 31 & Kaharudin & 33 & 1 & 33 & -0.47 & 0.22 \\
\hline 32 & M.Abdi & 34 & 1 & 33 & 0.52 & 0.28 \\
\hline 33 & Dhimas & 34 & 1 & 34 & 0.52 & 0.28 \\
\hline 34 & Eva & 32 & 1 & 32 & -1.47 & 2.1632 \\
\hline & $\begin{array}{r}\text { Sum } \\
\text { Average }\end{array}$ & 33.47058824 & 34 & 1138 & & 54.470 \\
\hline
\end{tabular}


DOI : https://doi.org/10.36456/tibuana.4.01.3175.1-6

Table 3. Determine the height of the back

\begin{tabular}{|c|c|c|c|c|c|c|}
\hline NO & NAME & BACK HEIGHT & $\mathrm{F}$ & $\mathrm{FX}$ & DEVIATION EACH SCORE (X) & $X^{2}$ \\
\hline 1 & Rindra & 61 & 1 & 61 & 0.52 & 0.28 \\
\hline 2 & M.Rizki & 62 & 1 & 62 & 1.52 & 2.33 \\
\hline 3 & Nanda & 63 & 1 & 63 & 2.52 & 6.39 \\
\hline 4 & Rida & 57 & 1 & 57 & -3.47 & 12.04 \\
\hline 5 & Alfin & 60 & 1 & 60 & -0.47 & 0.22 \\
\hline 6 & Septiyan & 61 & 1 & 61 & 0.52 & 0.28 \\
\hline 7 & Karto & 65 & 1 & 65 & 4,52 & 20,51 \\
\hline 8 & Fani & 61 & 1 & 61 & 0,52 & 0,28 \\
\hline 9 & Opik & 58 & 1 & 58 & -2.47 & 6.10 \\
\hline 10 & Eko & 61 & 1 & 61 & 0.52 & 0.28 \\
\hline 11 & Hendy & 60 & 1 & 60 & -0.47 & 0.22 \\
\hline 12 & Dennis & 61 & 1 & 61 & 0,52 & 0,28 \\
\hline 13 & Titin & 57 & 1 & 57 & -3.47 & 12.04 \\
\hline 14 & Dirga & 62 & 1 & 62 & 1.52 & 2.33 \\
\hline 15 & Akhmad & 62 & 1 & 62 & 1.52 & 2.33 \\
\hline 16 & M. Jafar & 61 & 1 & 61 & 0.52 & 0.28 \\
\hline 17 & M. Hanif & 61 & 1 & 61 & 0.52 & 0.28 \\
\hline 18 & Elsa & 54 & 1 & 54 & -6.47 & 41.86 \\
\hline 19 & Febian & 60 & 1 & 60 & -0.47 & 0.22 \\
\hline 20 & M.Abid & 63 & 1 & 63 & 2.52 & 6.39 \\
\hline 21 & Widodo & 61 & 1 & 61 & 0.52 & 0.28 \\
\hline 22 & Rahmatulloh & 59 & 1 & 59 & -1.47 & 2.16 \\
\hline 23 & Nova & 63 & 1 & 63 & 2.52 & 6.39 \\
\hline 24 & Candra & 60 & 1 & 60 & -0.47 & 0.22 \\
\hline 25 & Alex & 60 & 1 & 60 & -0.47 & 0.22 \\
\hline 26 & Prananda & 63 & 1 & 63 & 2.52 & 6.39 \\
\hline 27 & Gunawan & 58 & 1 & 58 & -2.47 & 6.10 \\
\hline 28 & Prayoga & 61 & 1 & 61 & 0.52 & 0.28 \\
\hline 29 & Rony & 60 & 1 & 60 & -0.47 & 0.22 \\
\hline 30 & Iwan & 60 & 1 & 60 & -0.47 & 0.22 \\
\hline 31 & Kaharudin & 60 & 1 & 60 & -0.47 & 0.22 \\
\hline 32 & M.Abdi & 61 & 1 & 61 & 0.52 & 0.28 \\
\hline 33 & Dhimas & 61 & 1 & 61 & 0.52 & 0.28 \\
\hline 34 & Eva & 59 & 1 & 59 & -1.47 & 2.16 \\
\hline & $\begin{array}{r}\text { Sum } \\
\text { Average }\end{array}$ & 60.47058824 & 34 & 2056 & & 140.47 \\
\hline
\end{tabular}



Table 4. Determine the length of the knee

\begin{tabular}{|c|c|c|c|c|c|c|}
\hline NO & NAME & KNEE LENGTH & $\mathrm{F}$ & FX & DEVIATION EACH SCORE (X) & $X^{2}$ \\
\hline 1 & Rindra & 56 & 1 & 56 & 4.29 & 18.43 \\
\hline 2 & M.Rizki & 57 & 1 & 57 & 5.29 & 28.02 \\
\hline 3 & Nanda & 51 & 1 & 51 & -0.70 & 0.49 \\
\hline 4 & Rida & 42 & 1 & 42 & -9.70 & 94.20 \\
\hline 5 & Alfin & 51 & 1 & 51 & -0.70 & 0.49 \\
\hline 6 & Septiyan & 55 & 1 & 55 & 3.29 & 10.85 \\
\hline 7 & Karto & 54 & 1 & 54 & 2.29 & 5.26 \\
\hline 8 & Fani & 54 & 1 & 54 & 2.29 & 5.26 \\
\hline 9 & Opik & 45 & 1 & 45 & -6.70 & 44.96 \\
\hline 10 & Eko & 53 & 1 & 53 & 1.29 & 1.67 \\
\hline 11 & Hendy & 50 & 1 & 50 & -1.70 & 2.91 \\
\hline 12 & Dennis & 53 & 1 & 53 & 1.29 & 1.67 \\
\hline 13 & Titin & 42 & 1 & 42 & -9.70 & 92.20 \\
\hline 14 & Dirga & 56 & 1 & 56 & 4.29 & 18.43 \\
\hline 15 & Akhmad & 58 & 1 & 58 & 6.29 & 39.61 \\
\hline 16 & M. Jafar & 55 & 1 & 55 & 3.29 & 10.85 \\
\hline 17 & M. Hanif & 53 & 1 & 53 & 1.29 & 1.67 \\
\hline 18 & Elsa & 35 & 1 & 35 & -16.70 & 279.08 \\
\hline 19 & Febian & 52 & 1 & 52 & 0.29 & 0.08 \\
\hline 20 & M.Abid & 60 & 1 & 60 & 8.29 & 68.79 \\
\hline 21 & Widodo & 47 & 1 & 47 & -4.70 & 22.14 \\
\hline 22 & Rahmatulloh & 49 & 1 & 49 & -2.70 & 7.32 \\
\hline 23 & Nova & 59 & 1 & 59 & 7.29 & 53.20 \\
\hline 24 & Candra & 51 & 1 & 51 & -0.70 & 0.49 \\
\hline 25 & Alex & 50 & 1 & 50 & -1.70 & 2.91 \\
\hline 26 & Prananda & 60 & 1 & 60 & 8.29 & 68.79 \\
\hline 27 & Gunawan & 45 & 1 & 45 & -6.70 & 44.96 \\
\hline 28 & Prayoga & 53 & 1 & 53 & 1.29 & 1.67 \\
\hline 29 & Rony & 51 & 1 & 51 & -0.70 & 0.49 \\
\hline 30 & Iwan & 52 & 1 & 52 & 0.29 & 0.08 \\
\hline 31 & Kaharudin & 52 & 1 & 52 & 0.29 & 0.08 \\
\hline 32 & M.Abdi & 55 & 1 & 55 & 3.29 & 10.85 \\
\hline 33 & Dhimas & 55 & 1 & 55 & 3.29 & 10.85 \\
\hline 34 & Eva & 47 & 1 & 47 & -4.70 & 22.14 \\
\hline & $\begin{array}{r}\text { Sum } \\
\text { Average }\end{array}$ & 51.70588235 & 34 & 1758 & & 973.05 \\
\hline
\end{tabular}

As a result, for Hips Width The size of the seat width used is $36 \mathrm{~cm}$ (95th percentile) so that large people can fit when occupying a chair, for Back Height The height of the chair is 57 $\mathrm{cm}$ (5th percentile) so that people with small body postures can sit comfortably without difficulty, for Knee Length The length of the knee is $61 \mathrm{~cm}$ (95th percentile).

\section{CONCLUSION}

Design of Flexible Chair College Have a result Hip Width size is $36 \mathrm{~cm}$, Back Height size is $57 \mathrm{~cm}$, and Knee Length is $61 \mathrm{~cm}$.

Measurement by anthropometry is carried out to achieve a comfortable, comfortable, safe, healthy condition for humans and of course also can create efficient working conditions with effective results or in other words, to achieve an ergonomic state.

\section{REFERENCE}

1. Wignjosoebroto, Sritomo. 2003. Pengantar Teknik 
TiBuana

Journal of applied Industrial Engineering-University of PGRI AdiBuana

DOI : https://doi.org/10.36456/tibuana.4.01.3175.1-6

p-ISSN 2622-2027

$e$-ISSN 2622-2035

ManajemenIndustri. GunaWidya.

Surabaya.

3. https://dokumen.tips/documents/makal

2. http://mylaporanti.blogspot.com/2015/

09/makalah-antropometri-dengan-

metode.html

(Diakses

15

desember2019, 19:19)

ah-antropometri.html (Diakses 15 desember 2019, 20:33)

4. https://antropometriindonesia.org/inde x.php/detail/sub/2/7/0/pengantar_antr opometri (Diakses 15 desember 2019, $20: 36$. 